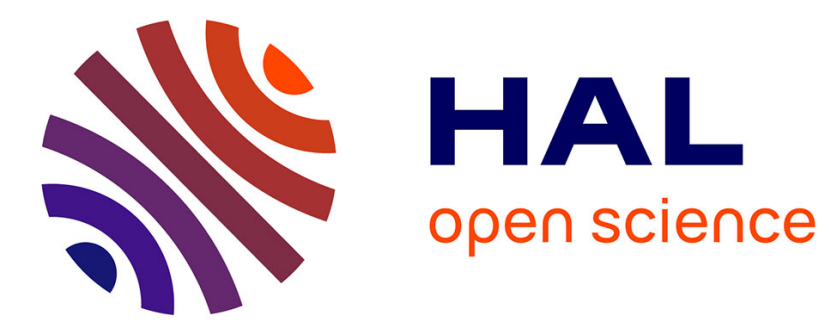

\title{
Détourer la mémoire
}

Patrick Boucheron

\section{To cite this version:}

Patrick Boucheron. Détourer la mémoire. La mémoire nous joue-t-elle des tours?, Presses Universitaires de France, pp.221-220, 2018, 10.3917/puf.frydm.2018.01.0215 . hal-03264930

\section{HAL Id: hal-03264930 \\ https://hal.science/hal-03264930}

Submitted on 24 Jun 2021

HAL is a multi-disciplinary open access archive for the deposit and dissemination of scientific research documents, whether they are published or not. The documents may come from teaching and research institutions in France or abroad, or from public or private research centers.
L'archive ouverte pluridisciplinaire HAL, est destinée au dépôt et à la diffusion de documents scientifiques de niveau recherche, publiés ou non, émanant des établissements d'enseignement et de recherche français ou étrangers, des laboratoires publics ou privés. 


\section{DÉTOURER LA MÉMOIRE}

\section{Patrick Boucheron}

in René Frydman et al., La mémoire nous joue-t-elle des tours?

Presses Universitaires de France | « Hors collection »

2018 | pages 221 à 220

ISBN 9782130734680

Article disponible en ligne à l'adresse :

https://www.cairn.info/la-memoire-nous-joue-t-elle-des-tours---page-221.htm

Distribution électronique Cairn.info pour Presses Universitaires de France.

(C) Presses Universitaires de France. Tous droits réservés pour tous pays.

La reproduction ou représentation de cet article, notamment par photocopie, n'est autorisée que dans les limites des conditions générales d'utilisation du site ou, le cas échéant, des conditions générales de la licence souscrite par votre établissement. Toute autre reproduction ou représentation, en tout ou partie, sous quelque forme et de quelque manière que ce soit, est interdite sauf accord préalable et écrit de l'éditeur, en dehors des cas prévus par la législation en vigueur en France. Il est précisé que son stockage dans une base de données est également interdit. 


\title{
Détourer la mémoire
}

\author{
Patrick Boucheron
}

La société ne se souvient de rien.

Maurice Halbwachs entre Henri Bergson et Marc Bloch

\section{$\mathrm{L}$}

a mémoire pour les historiens ? Elle existe et elle n'existe pas. Elle existe, puisqu'ils s'échinent le plus souvent - et notamment depuis les années 1980 - à défendre la méthode historique contre cette folle du logis, thématisant une opposition farouche entre rigueurs historiennes et effervescences mémorielles. Elle n'existe pas, car au-delà du tremblé du souvenir des témoins et de la méfiance qu'en bonne rigueur on doit leur opposer, au-delà du travail actif des entrepreneurs de mémoire et des politiques d'oubli ou de commémoration, au-delà même de la production documentaire et de la présence monumentale, une société, d'elle-même, ne se souvient de rien - sauf à considérer qu'il existe une psyché collective, ce que nul ou presque n'est prêt à admettre.

Cette position a longtemps prévalu chez les philosophes et les psychologues, notamment parce qu'elle pouvait s'autoriser de la gloire d'un grand nom : celle d'Henri Bergson ${ }^{1}$. On sait quelle influence eût son maître livre Matière et mémoire (1896) sur les sciences sociales naissantes, mais aussi sur la littérature, et l'on sait également combien la philosophie bergsonienne du temps peut être envisagée comme une vigoureuse défense et illustration de l'idée de nouveauté, ou plus

1. François Azouvi, La Gloire de Bergson. Essai sur le magistère philosophique, Paris, Gallimard, 2007. 


\section{La mémoire nous joue-t-elle des tours?}

exactement de la possibilité que du radicalement neuf fasse irruption dans la durée ${ }^{1}$.

Pour lui, le passé se survit à lui-même sous deux formes distinctes, qu'il appelle respectivement "mémoire-habitude» et "mémoire pure ». La première, adaptée au présent, consiste à mobiliser des ressources psychologiques pour agir (pour agir concrètement, soit pour Bergson agir sur la matière). Elle est donc communicable, car nous agissons collectivement. À l'inverse, la mémoire pure est une accumulation de souvenirs sédimentés, d'images de la vie psychique, propre à l'individu. Pour les atteindre, l'individu doit s'éloigner de la société, se retirer.

De Bergson, son maître, Maurice Halbwachs s'inspirait pour travailler à une forme d'introspection prudente - mais il le faisait à la lumière des leçons de son autre maître, Émile Durkheim, qui lui enseignait la nécessité de l'examen critique systématique. Voici pourquoi, dans Les Cadres sociaux de la mémoire, paru en 1925, il établit la mémoire comme fait social. Pour aller vite, un souvenir est une reconstruction du passé, la recomposition d'une image ancienne - même lorsqu'on se souvient individuellement, on le fait en fonction du système de représentations présent au moment où l'on se souvient, et ce système de représentations est celui d'une société donnée à un moment donné. C'est cela qu'on peut appeler les cadres sociaux de la mémoire - dont le premier est le langage lui-même - car cette mise en présence du passé, "s'accorde à chaque époque avec les pensées dominantes de la société ${ }^{2} »$.

Dans une lettre datée du 27 avril 1926, belle lettre d'incompréhension, de ces incompréhensions fécondes qui font le nerf des échanges intellectuels, Bergson lui en fait reproche: "Je me demande si vous n'êtes pas allé trop loin dans la résolution de l'individuel au collectif, et je dois dire que même après avoir fait le plus grand effort pour me

1. Voir Henri Bergson, Histoire de l'idée de temps. Cours au Collège de France (1902-1903), Camille Riquier (éd.), Paris, Puf, 2016.

2. Maurice Halbwachs, Les Cadres sociaux de la mémoire (1925), Paris, Albin Michel, 1994, p. VIII. 


\section{Détourer la mémoire}

placer à votre point de vue, je n'arrive pas à reporter sur la société ce qu'il y a de spécifique et par conséquent d'essentiel, dans la conservation et l'évocation du souvenir ${ }^{1}$. » Or voici où je veux en venir : ce qui est trop pour Henri Bergson n'est pas assez pour Marc Bloch, qui fait paraître en 1925 dans la Revue de synthèse historique un compte rendu critique du livre de Maurice Halbwachs. Il en retient une leçon essentielle: "La société n'interprète ou même ne connaît le passé qu'à travers le présent et par ailleurs le présent n'a pour elle de sens concret et de valeur émotionnelle que parce que derrière lui s'entrevoit une certaine durée ${ }^{2}$.»

Mais pour l'historien, le chemin n'est qu'à demi parcouru. Bloch reproche en effet à Halbwachs de n'avoir pas été jusqu'au bout de son entreprise théorique, qui consiste à arracher la notion de mémoire aux psychologues pour la soumettre au social. Il remarque que Halbwachs, comme tous les durkheimiens d'alors, se contente d'appliquer l'épithète collectif à des phénomènes qu'il pense en fait comme individuels: "Je serais assez tenté d'en tenir pour responsable le vocabulaire durkheimien, caractérisé par l'emploi, avec l'épithète "collectif", de termes empruntés à la psychologie individuelle $^{3}$.» La mise en garde a été entendue par Maurice Halbwachs, qui tient compte des critiques de Marc Bloch dans ses écrits ultérieurs, plus tard réunis dans l'ouvrage posthume intitulé La Mémoire collective 4 . Pour aller vite, le souvenir individuel n'y apparaît plus seulement qu'en tant qu'il s’identifie à un élément de la mémoire collective, mais interagit avec lui par une dynamique d'aller-retour qui tient compte de la diversité des mémoires collectives. Autrement

1. Citée par Marie Jaisson, "Mémoire collective et espace social», in Maurice Halbwachs, La Topographie légendaire des évangiles en Terre sainte. Étude de mémoire collective (1941), Paris, Puf, 2008, p. 73-97 (p. 79).

2. Marc Bloch, "Mémoire collective, tradition et coutume. À propos d'un livre récent ", Revue de synthèse historique, $\mathrm{n}^{\circ} 14,1925$, p. 73-83 (p. 76).

3. Ibid., p. 78.

4. Maurice Halbwachs, La Mémoire collective, Paris, 1955. Sur l'évolution problématique qui mène des Cadres sociaux à La Mémoire collective, voir la postface de l'édition critique établie par Gérard Namer, Paris, Albin Michel, 1997, notamment p. 282 et $s$. 
dit, comme l'écrit Paul Ricœur, «le pas franchi dans La Mémoire collective consiste à désimpliquer la référence à la mémoire collective du travail même de la mémoire personnelle en train de rappeler ses souvenirs ${ }^{1} »$.

Or la réflexion du sociologue sur les usages sociaux de la mémoire culmine dans un livre proprement génial, paru en 1941 dans une indifférence presque absolue, La Topographie légendaire des évangiles en Terre sainte. Immanquablement, le thème de la mémoire guidait les pas d'Halbwachs vers l'étude d'un espace urbain dramatiquement clivé, dont l'unité se brise sous le poids de souvenirs antagonistes. La question de la mémoire et de ses remplois sociaux rencontrait celle des territoires, et de la ville, où le passé est toujours un présent en glissement: "C'est l'image seule de l'espace qui, en raison de sa stabilité, nous donne l'illusion de ne point changer à travers le temps et de retrouver le passé dans le présent; mais c'est bien ainsi qu'on peut définir la mémoire ${ }^{2}$.»

De quoi s'agit-il ? Maurice Halbwachs avait choisi de placer ses pas dans ceux du Pèlerin de Bordeaux, le premier dont l'histoire ait enregistré l'itinéraire, dans le premier quart du IV siècle. Il chemine, il décrit et il invente - il localise dans la ville le souvenir biblique, en recouvrant la mémoire juive. Le sociologue le suit donc, pour partir en quête du mouvement même de la construction mémorielle et comprendre la manière dont les souvenirs viennent moins des choses du passé que des besoins du présent. Mais ce qu'il rencontre en chemin, c'est bien ce qu'il appelle la « résistance des choses », leur obstination têtue et sonore. Il y a des accidents de terrain, des pentes, des anfractuosités: le souvenir s'y accroche et, dès lors, n'est plus si aisément manipulable. Les pierres sont comme des obstacles dans le cours d'un fleuve. Des souvenirs cheminant séparément, et provenant de sources différentes, peuvent confluer, y mêler leurs eaux (ainsi dans les récits légendaires enrobant la colline de Sion); d'autres au contraire, but-

1. Paul Ricœur, La Mémoire, l'histoire, l'oubli, Paris, Seuil, 2000, p. 147.

2. Maurice Halbwachs, La Mémoire collective, op. cit., p. 236. 


\section{Détourer la mémoire}

tant sur ces rochers, s'y séparent et s'y ralentissent, s'épanchant sur une surface où peut se mirer une narration scandée (comme c'est le cas à propos du saint Sépulcre). Mais dans tous les cas, il n’y a pas de lieux de mémoire, car le monument n'est rien d'autre que la solidification desséchée d'un souvenir. Ce qu'il y a: des circulations, des réseaux, des correspondances.

Le livre n'a commencé à être lu que dans les années 1990, lorsque les sciences sociales se sont emparées du fait urbain, à partir notamment de la problématique de la trace. Bernard Lepetit, historien des villes modernes, s'en est fait le commentateur subtil. Il a montré que la réaffectation permanente des traces du passé au présent de la ville, c'est-à-dire la manière dont la ville, dans ses formes matérielles, accumule des souvenirs, des fragments disjoints de passés différents que l'on vit toujours en contemporanéité produit une analogie entre la forma urbis et la forma mentis fascinante mais trompeuse ${ }^{1}$. La ville, en somme, comme un organisme vivant, est moins stable que stationnaire. Car le temps passant, il impose sans cesse des remaniements. Dans l'un des textes qui accompagnent la réédition critique du livre de Maurice Halbwachs en 2008, Éric Brian a montré l'importance de ce concept clé de remaniements chez Maurice Halbwachs, qui vaut pour les structures matérielles comme pour les cadres mémoriels d'une société. Car le remaniement des lieux et des traditions passe toujours par trois phases: le réinvestissement (se remémorer, c'est toujours réoccuper les lieux), la transaction (il faut alors s'accorder sur les interprétations concurrentes des lieux), la consolidation - et c'est ainsi que se transmet une tradition, ainsi qu'une mémoire perdure, non pas en dépit mais du fait même de ses transformations ${ }^{2}$.

C'est donc bien Maurice Halbwachs, sociologue des cadres sociaux de la mémoire confrontée à la matérialité têtue des formes

1. Bernard Lepetit, "Le présent de l'histoire ", in Carnet de croquis. Sur la connaissance historique, Paris, Albin Michel, 1999, p. 252-283 (p. 276).

2. Éric Brian, "Portée du lexique halbwachsien de la mémoire», dans Maurice Halbwachs, La Topographie légendaire des évangiles en Terre sainte, op. cit., p. 113146, notamment p. 125 et s. 


\section{La mémoire nous joue-t-elle des tours?}

urbaines, qui peut permettre aujourd'hui aux historiens de résoudre leur dilemme face à la mémoire, au «je sais bien mais quand même» qui constitue l'une des expressions les plus désarmantes du déni ${ }^{1}$. La société ne se souvient de rien, mais chacun y adapte en permanence le passé aux exigences du présent - et c'est cela que l'on peut appeler mémoire collective. «Seulement, dans cet effort d'adaptation, les hommes se heurtent à la résistance des choses, parfois des rites, des formules, qui sont mécaniques, matériels, ici des commémorations anciennes, fixées dans des pierres, des églises, des monuments, où les croyances et les témoignages d'autrefois ont pris forme d'objets solides et durables. Il est vrai que ces objets, tels qu'ils nous apparaissent, résultent eux-mêmes d'une adaptation antérieure des croyances héritées du passé aux croyances du présent, et, en même temps, de celles-ci aux vestiges matériels des croyances anciennes. On remonte ainsi le cours du temps. Mais, quelque époque qu'on envisage, l'attention se dirige non vers l'origine de tout ce développement, mais vers les groupes de fidèles, vers les œuvres de commémoration $^{2}$.»

1. Octave Mannoni, Clefs pour l'imaginaire ou l'Autre Scène, Paris, Seuil, 1969.

2. Maurice Halbwachs, La Topographie légendaire des évangiles en Terre sainte, op. cit., p. 163. 